\title{
A Spatio-Temporal Distribution and Dynamics of Major-, Minor- and Trace- Elements in Two Temperate Urban Lacustrine Ecosystems of Srinagar, Kashmir
}

\author{
Umar Nazir Bhat* and Anisa Basheer Khan \\ Department of Ecology and Environmental Sciences, Pondicherry University, Puducherry, 605014, India \\ Email: unbspn@gmail.com
}

\begin{abstract}
Water bodies act as indicators of the extent of anthropogenic interference and pressure while evolving exhibitive fluxes in intrinsic homeostasis. The ecological assessment of standard biophysicochemical variables through space-time monitoring in the region of anthro-urban intensification identifies such ecosystem responses. Sampling, preservation, preparation and analysis follow typical procedures for all the representative testers. Statistical procedures for inference of results involve Mean, Standard Deviation, Range, Correlation, Regression, Analysis of Variance (ANOVA), Cluster Analysis and Principal Components Analysis (PCA). Temperature, pH, conductivity and ionic composition of the surface waters have $\mathrm{p}<0.01$ and $\mathrm{R}^{2}>0.5$. Total $\mathrm{P}$ perimeter outdid critical eutrophic index $\left(\leqslant 0.05 \mathrm{mgL}^{-1}\right)$ but nitrate $\mathrm{N}$ persisted underneath it $\left(\leqslant 0.5 \mathrm{mgL}^{-1}\right)$ ordinarily. Elevated nutrient levels reflect multiple human origins and catchment interventions. Priority pollutants (As, $\mathrm{Cd}, \mathrm{Cr}, \mathrm{Cu}, \mathrm{Pb}, \mathrm{Ni}$ and $\mathrm{Se}$ ) remained below chronic levels with exception of $\mathrm{Fe}$ and $\mathrm{Zn}$ exceeding maximum permissible limits for irrigation purpose of the water. Major elements in surface sediments are $\mathrm{Si}>\mathrm{Ca}>\mathrm{Mg}>\mathrm{K}>\mathrm{Na}>\mathrm{P}>\mathrm{S}>\mathrm{Cl}$ besides the micro and trace ones follow as $\mathrm{Fe}>\mathrm{Al}>\mathrm{Zn}>\mathrm{Mn}>\mathrm{Cu}>\mathrm{Cr}>\mathrm{Ni}>\mathrm{Co}>\mathrm{As}>\mathrm{Sn}>\mathrm{Pb}>\mathrm{Cd}$. Sediments correspond to calcite catchment character to conform their sink-selves. The peak biomass values as $\mathrm{gm}^{-2}$ are 880.2 in case of Myriophyllum aquaticum, 678.4 in Nelumbo nucifera, 182.4 in Ceratophyllum demersum and 45 in Salvinia natans. The upgraded biomass configuration coincides with supplementary bioconcentration. $\mathrm{C} / \mathrm{N}<10$ implies low decomposition rates and autochthonous origin of organic matter. A semidrainage type of flushing hydrology ensures low basin holding time for self-reclaimed nutrient balance. Sediment dredging is beneficial for internal nutrient deloading only after external inputs are curtailed. Biological scavenging and biogenic calcification together lower the bioavailability of nutrients and toxics. Hence, the systemic insitu pliability can prevail till exsitu lacustrine management fortifies euoecism to evade detrimental dysoecism.
\end{abstract}

Keywords: Sediment chemistry, productivity, organic matter, trophic status, trace-elements.

\section{Introduction}

Lakes are natural or artificial, brackish or freshwater lentic impoundments located within inland geologic depressions of Earth's crust. Their ephemeral feature manifests in originating due to certain geological processes and terminating as a result of disrupted hydrological balance or sedimentation [1]. Lacustrine ecosystems possess a dynamic equilibrium among and between physical, chemical and biological attributes responding to natural and anthropogenic fluctuations. The transition from oligotrophy to eutrophy steered by nutrient enrichment, sedimentation and higher productivity eventually transforms a lake into dystrophic marsh [2]. The diversified anthropogenic activities have led to the global sprawl of spectra of contaminants intoxicating various environmental compartments and posing potential risks for biota and human health as well [3] and [4]. Consequently, the natural setting and homeostasis of aquatic ecosystems is continuously and significantly altered by progressive socioeconomic, urban, industrial and agricultural developments [5], [6] and [7]. The impacts of the human activities on the overall surfacewater quality devalue its suitability for a designated purpose besides influencing the structure and function of its abiotic and biotic subsystems [8]. Both the eutrophication process and water quality classification in lakes is subject to quantum of principal chemical constituents and pollutants distributed within the water column, sediment and biota. Water quality [9], sediment characteristics [10], biological 
indicators [11] and even key ecological processes like primary production are essential to assess the integrity and health of the ecosystem [12]. The temporal variation in ionic composition of natural waters along with its spatial distribution in a region aids significantly to distinguish the source [13]. It is incumbent to conduct continuous field monitoring studies to understand the characterisation and contribution of each contaminant responsible for environmental deterioration to evolve sustainable ecoconservation strategies, practices and technologies [14], [15] and [16]. Therefore, in order to understand the level of contamination and innate capacity of the lake components to dissipate it for wellbeing of the ecosystem is undertaken for finding provisions of prospective ecomanagement approaches.

\section{Materials and Methods}

\subsection{Study Area}

Water, sediment and macrophytes from 50 sampling stations of five study sites of Anchar and Dal lakes (Fig. 1) were collected from March, 2011 to February, 2013. The five sites designated as A1, A2, D1, D2 and D3 were each further fragmented into ten sampling stations in order to obtain composite representative samples in triplicate for enhanced precision. Due consideration is given to collect and preserve the sub-samples of water, sediment and hydrophytes from the same spot.

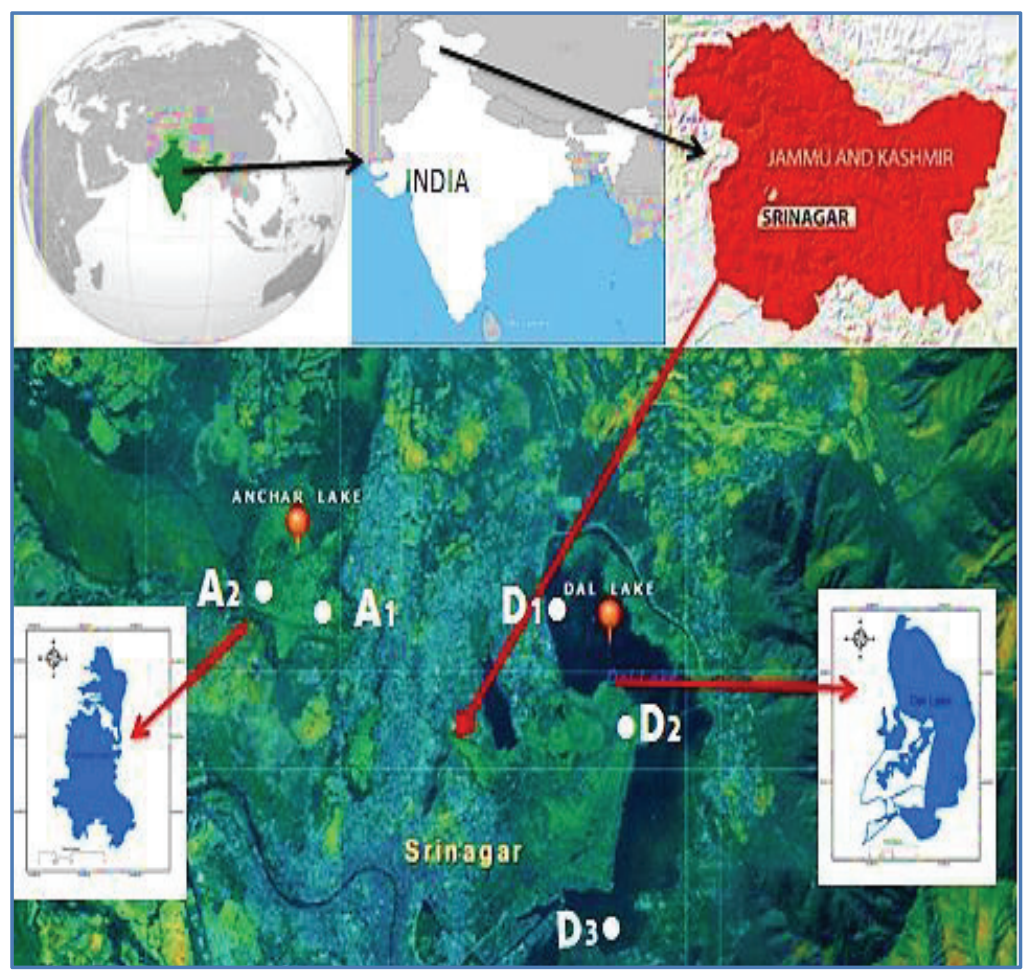

Figure 1. Location of study area.

\subsection{Physico-chemical Analysis of Water}

The water samples were analysed for temperature, $\mathrm{pH}$, conductivity, bicarbonates, chloride, calcium, magnesium, sodium, potassium, nitrate nitrogen and total phosphorus on monthly basis while trace elements of $\mathrm{Al}, \mathrm{As}, \mathrm{Cd}, \mathrm{Co}, \mathrm{Cr}, \mathrm{Cu}, \mathrm{Fe}, \mathrm{Hg}, \mathrm{Mn}, \mathrm{Ni}, \mathrm{Pb}, \mathrm{Se}$, Sn and $\mathrm{Zn}$ were measured for spring, summer and winter seasons. The collection, preservation, preparation, storage and estimation of water samples follow standard methods [17], [18], [19], [20], [21] and [22]. 


\subsection{Chemical Analysis of Sediments}

The sediment analysis includes $\mathrm{H}$-ion concentration, electrical conductivity, bicarbonates, organic-C, organic matter, total-N, C/N ratio, elemental composition of $\mathrm{Ca}, \mathrm{Cl}, \mathrm{K}, \mathrm{Mg}, \mathrm{Na}, \mathrm{P}, \mathrm{S}, \mathrm{Si}$ and the given trace elements for each season. Electrical Conductivity is determined from filtrate of $1: 2 \mathrm{~W} / \mathrm{V}$ sediment- distilled water suspension using a standard $0.01 \mathrm{M} \mathrm{KCl}$ solution at room temperature. For $\mathrm{HCO}_{3}{ }^{-} 20 \mathrm{~mL}$ of the filtered extract is titrated against $0.02 \mathrm{~N} \mathrm{H}_{2} \mathrm{SO}_{4}$ thrice to obtain concordant anion(s) concentration. Organic $\mathrm{C}$ and total $\mathrm{N}$ is estimated respectively by Walkley and Black wet oxidation method and Kjeldahl method.

\subsection{Bio-chemical Analysis of Macrophytes}

The macrophytic biomass of the emergent M. aquaticum (M.a.); the rooted floating $N$. nucifera (N.n.); the submerged C. demersum (C.d.) and the free floating S. natans (S.n.) were analysed for total chlorophyll content, biomass, productivity, specific growth rate, mineral composition $(\mathrm{Ca}, \mathrm{Cl}, \mathrm{K}, \mathrm{Mg}, \mathrm{N}$, $\mathrm{Na}, \mathrm{P}, \mathrm{S}, \mathrm{Si}$ ) and the trace elements during their sprouting (sp.)-(March to May), peak growth (pg.)(June to October) and senescence (sn.)-(November to February) phases. For plant and sediment samples various parametric evaluations are performed according to the protocol [23], [24] and [25]. The multi-elemental quantification of $\mathrm{Ca}, \mathrm{Cl}, \mathrm{K}, \mathrm{Mg}, \mathrm{Na}, \mathrm{P}, \mathrm{S}$ and $\mathrm{Si}$ in sediments and plant biomass is performed using Wavelength Dispersive X-ray Fluorescence Spectrometer (WD-XRF) at Central Instrumentation Facility (CIF), Pondicherry University. However, the trace elements in all samples were determined at Sophisticated Analytical Instrument Facility (SAIF) IITM, Chennai using ICP-OES (Perkin Elmer Optima 5300 DV) instrument.

\subsection{Statistical Methods}

The descriptive and illustrative statistical analysis including Mean, Standard Deviation, Range, Correlation, Regression, one-way ANOVA, Cluster Analysis and Principal Components Analysis (PCA) is performed using statistical software of MS-Excel 2010, Origin 8.5, PAST 3 and SPSS 19.

\section{Results}

The results of the study establish interdependent and interactive compartmentalization of inorganic elements in lacustrine components of water, sediment and macrophytes on space and time scales.

\subsection{Surface Waters}

The inter-annual monthly quantitative analysis (average values) of water quality parameters present a vivid inconsistency. The mean surface water temperatures recorded for the five study sites from March 2011 to February 2013 reflect a distinct contrast with higher values in summers and a declining trend towards the winters as a season specific feature. It ranged between 5 to $30^{\circ} \mathrm{C}$ with averages of $18.6 \pm 7.3$, $17.9 \pm 7.2,18.1 \pm 7.4,18.1 \pm 7.0,18.5 \pm 7.0$ recorded at the five sites. The temperature variance between sites $(\mathrm{F}=.013$ and $\mathrm{p}>0.05)$ is insignificant, however, seasonal variation is significant $(\mathrm{F}=1.39$ and $\mathrm{p}<$ 0.05). The statistical insignificance in recorded epilimnion temperature variance as well as significant temporal discrepancy discloses similar geo-climatic setting of the lakes. $\mathrm{pH}$ fluctuated between 7.2 and 8.8 symbolizing alkaline waters with comparatively higher summer peaks established by macrophytic productivity. The site-wise averages are $8.4 \pm 0.32,8.5 \pm 0.28,8.2 \pm 0.43,8.1 \pm 0.43$ and $8.0 \pm 0.41$. ANOVA for $\mathrm{pH}$ has significance between lakes $(\mathrm{F}=7.807$ and $\mathrm{p}<0.05)$ and between seasons $(\mathrm{F}=9.782$ and $\mathrm{p}<$ $0.05)$ as well. Although the entire recorded $\mathrm{pH}$ profile $(>7)$ reflects buffered waters but has prominent variance both site- and time-wise suggesting position and period specific photosynthetic activity that withdraws $\mathrm{HCO}_{3}{ }^{-}$thereby elevating diurnal $\mathrm{pH}$. Conductivity exhibited a fall towards the spring and summer months presumably due to dilution and ion uptake by flora present in the water-column. The conductivity range observed has a minimum value of $100 \mu \mathrm{S} / \mathrm{Cm}$ and a maximum of $565 \mu \mathrm{S} / \mathrm{Cm}$ besides having average site values of $353.9 \pm 57.46,306.4 \pm 48.88,294.7 \pm 113.83,266.8 \pm 100.87$ and $271.3 \pm 87.60$. Seasonal ANOVA of conductivity is more significant $(\mathrm{F}=7.829$ and $\mathrm{p}<0.05)$ than lake comparisons 
$(\mathrm{F}=4.526$ and $\mathrm{p}<0.05)$. The diminishing conductivity at improved temperature conditions suggest higher bioaccumulation rate of nutrient ions like $\mathrm{HCO}_{3}^{-}, \mathrm{Ca}, \mathrm{Mg}, \mathrm{Na}, \mathrm{K}, \mathrm{P}$. The examination of the correlation matrix verily suggests temperature increments elevating $\mathrm{pH}$ but diminishing conductivity, $\mathrm{HCO}_{3}{ }^{-}, \mathrm{Ca}, \mathrm{Mg}, \mathrm{Na}, \mathrm{K}$ and $\mathrm{P}$ significantly. However, $\mathrm{Cl}$ and $\mathrm{NO}_{3}-\mathrm{N}$ remain unaffected due to temperature and $\mathrm{pH}$ ordeal. The water characteristics of bicarbonates, chloride, calcium, magnesium, sodium, potassium, nitrate nitrogen and total phosphorus although differ in being as major $(>5 \mathrm{mg} / \mathrm{L})$ or minor $(0.01$ to $10 \mathrm{mg} / \mathrm{L}$ ) elements but show almost an identical scenario of retreat during peak growth phases of macrophytes and again pickup in post senescence period. The PCA results reveal that variables of conductivity, $\mathrm{K}, \mathrm{Na}, \mathrm{Mg}, \mathrm{Ca}, \mathrm{P}, \mathrm{HCO}_{3}^{-}, \mathrm{NO}_{3}-\mathrm{N}$ and $\mathrm{Cl}$ contribute more on $\mathrm{PC} 1(60.27 \%)$ whereas temperature and $\mathrm{pH}$ predominate on $\mathrm{PC} 2(28.73 \%)$. An equation with the world average stream content $\left(\mathrm{mgL}^{-1}\right)$ of recorded $\mathrm{Ca}(>15), \mathrm{Mg}(>4), \mathrm{K}(>2.3)$ and $\mathrm{Na}(>6.3)$ acclaim pronounced human inputs from common uses like fertilizers, plaster, pigments, lime, alloys, pharmaceuticals, batteries, food additives, glass, baking powder, soft drinks, electroplating, caustic soda, water treatment chemicals, etc. The $\mathrm{HCO}_{3}{ }^{-}$concentration ranges from 60 to $218 \mathrm{mg} / \mathrm{L}$ and at the selected sites average to $126.2 \pm 42.57$, $121.8 \pm 41.77,105.2 \pm 24.93,105.0 \pm 25.89$ and $105.7 \pm 27.95$ respectively. Bicarbonates register significant seasonal variation $(\mathrm{F}=35.431$ and $\mathrm{p}<0.05)$ only. The waters are well buffered having insignificant site variation but there is gradual increase in summers due to higher proton uptake during peak photosynthetic activity thereby shifting $\mathrm{CO}_{2}$ and $\mathrm{HCO}_{3}$ equilibrium. Contrarily, lower $\mathrm{pH}$ during winter supposedly on account of excess carbonic acid generation is due to higher [Respiration : Photosynthesis] proportion. Similarly, The ANOVA classifies other variables as significantly fluctuating between sites (p $<0.05$ ) except $\mathrm{Ca}$ but insignificantly varying between seasons ( $\mathrm{p}>0.05$ ) except $\mathrm{Ca}, \mathrm{Mg}$ and $\mathrm{K}$. The respective temporal range and site averages for these elements are presented in Table 1. ANOVA reveals significant seasonal variance in temperature, $\mathrm{pH}$, conductivity and $\mathrm{BIC}$ compared to significant site contrasts for $\mathrm{Cl}, \mathrm{Mg}, \mathrm{Na}, \mathrm{K}, \mathrm{NO}_{3}-\mathrm{N}$ and $\mathrm{P}$. The bicarbonates, chloride, calcium, magnesium, sodium, potassium, nitrate nitrogen and total phosphorus show almost an identical scenario of retreat during peak growth phases of macrophytes and again pickup in post senescence period. The comparative results exhibit least inter se difference in temperature, $\mathrm{pH}, \mathrm{Na}, \mathrm{K}$, bicarbonates, $\mathrm{Cl}, \mathrm{Mg}$ but larger fluctuations in other parameters of $\mathrm{NO}_{3}-\mathrm{N}, \mathrm{P}, \mathrm{Ca}$ and conductivity. The similarity index Fig.2 of the sites in form of a dendrogram derived from hierarchical cluster analysis of water variables suggests a site specific peculiarity dependent on micro local conditions and perturbation types existing in the lakes. The correlation matrix provided in Table 2 shows Pearsons correlation coefficients and statistical significance with positive and negative significantly related water quality variables in bold asterisks. The regression analysis of water variables along with regression coefficients given in Fig.3 portrays vivid intra-aqueous interdependence by describing line of best-fit $\left(\mathrm{R}^{2}\right)$. The correlation matrix and regression analysis of water variables reflected in Table 2 and Fig. 3 deduce a strong positive correlation between temperature and $\mathrm{pH}\left(\mathrm{p}<0.01, \mathrm{R}^{2}=0.8617\right)$; temperature and conductivity $\left(\mathrm{p}<0.01, \mathrm{R}^{2}=0.8419\right)$; temperature or conductivity with other ions and intra-aqueous ionic composition $\left(\mathrm{p}<0.01, \mathrm{R}^{2}>0.5\right)$. The supportive understanding of water variable interaction is offered by significant coefficient of determination $\left(\mathrm{R}^{2}\right)$ alongside simple least square regression lines for $\mathrm{pH}$ versus temperature $(0.8)$, conductivity versus $\mathrm{pH}$ (0.9), conductivity versus temperature (0.8) and BIC versus temperature (0.5). The graphical presentation in Fig.4 illustrated average trace element concentration in lake waters and their seasonal changes. The cationic content revealed a progression order of $\mathrm{Ca}>\mathrm{Mg}>\mathrm{Na}>\mathrm{K}$ all along with anionic predominance of $\mathrm{HCO}_{3}{ }^{-}$and $\mathrm{Cl}^{-}$. The overall total $\mathrm{P}$ limit surpassed critical eutrophic index $(\leqslant 0.05$ $\left.\mathrm{mgL}^{-1}\right)$ but nitrate $\mathrm{N}$ persisted underneath it $\left(\leqslant 0.5 \mathrm{mgL}^{-1}\right)$ mostly. $\mathrm{NO}_{3}-\mathrm{N}$ shows the only negative association with $\mathrm{HCO}_{3}{ }^{-}$concluding eutrophication steers on $\mathrm{C}$ assimilation for it serves as the main inorganic $\mathrm{N}$ source. The close covariance of $\mathrm{P}$ with $\mathrm{Ca}, \mathrm{Mg}, \mathrm{Na}$ and $\mathrm{K}$ depict their analogous limited absorbance constraint by the biota. The trace elements do not follow any definite seasonal variability. But ANOVA between sites confirm significance for $\mathrm{Al}(\mathrm{F}=49.14$ and $\mathrm{p}<0.05), \mathrm{Cr}(\mathrm{F}=3.854$ and $\mathrm{p}$ $<0.05), \mathrm{Cu}(\mathrm{F}=28.64$ and $\mathrm{p}<0.05)$ and $\mathrm{Ni}(\mathrm{F}=7.05$ and $\mathrm{p}<0.05)$. Besides as per Table $3, \mathrm{Al}$ shows significant correlations with $\mathrm{Co}, \mathrm{Cu}$ and $\mathrm{Ni}$ while as association of As is significant with $\mathrm{Cd}$ and $\mathrm{Ni}$. While comparing the observed average epilimnion trace element concentration $\left(\mathrm{\mu gL}^{-1}\right)$ with the average global stream abundance in absence of any baseline data, a vivid skewed hike for As $(\sim 10>2)$, Cd $(\sim$ $3>1), \mathrm{Cr}(\sim 40>1)$, $\mathrm{Co}(\sim 10>0.2), \mathrm{Cu}(\sim 130>10), \mathrm{Fe}(\sim 5600>700), \mathrm{Pb}(\sim 90>3), \mathrm{Mn}(\sim 600>$ 7), Ni $(\sim 30>1)$, Se $(\sim 9>0.2)$, Sn $(\sim 190>0.1)$ and $\mathrm{Zn}(\sim 18520>20)$ proclaim their anthropogenic geneses. The exception of $\mathrm{Fe}\left(>5 \mathrm{mgL}^{-1}\right)$ and $\mathrm{Zn}\left(>2 \mathrm{mgL}^{-1}\right)$ enhance the maximal concentrations in 
irrigation recommendations. Still, $\mathrm{Zn}$ has lesser toxicity in organic soils at $\mathrm{pH}>6$ and Fe contributes towards acidification and reduced $\mathrm{P}$ availability. $\mathrm{Al}$ (a non-priority pollutant) at $\mathrm{pH} 6.5$ to 9 is well below the freshwater Criterion Continuous Concentration (CCC) chronic exposure limit of $\geqslant 87 \mu \mathrm{gL}^{-1}$. $\mathrm{Fe}$ exceeds $\mathrm{CCC}$ at $>1000 \mu \mathrm{gL}^{-1}$ too. But all the priority pollutants of $\mathrm{As}, \mathrm{Cd}, \mathrm{Cr}, \mathrm{Cu}, \mathrm{Pb}, \mathrm{Ni}$ and $\mathrm{Se}$ remained below chronic level of CCC except $\mathrm{Zn}$.

Table 1. Temporal range and site averages for elements in surface waters

\begin{tabular}{ccccccc}
\hline ELEMENT & RANGE & A1 & A2 & D1 & D2 & D3 \\
\hline $\mathbf{C l}(\mathbf{m g} / \mathbf{L})$ & $11.6-28.9$ & $20.1 \pm 3.40$ & $17.0 \pm 1.92$ & $17.1 \pm 3.06$ & $21.4 \pm 3.07$ & $16.3 \pm 1.44$ \\
$\mathbf{C a}(\mathbf{m g} / \mathbf{L})$ & $38-102$ & $88.1 \pm 8.39$ & $86.2 \pm 7.56$ & $75.1 \pm 17.34$ & $75.6 \pm 17.09$ & $71.4 \pm 16.2$ \\
$\mathbf{M g}(\mathbf{m g} / \mathbf{L})$ & $16.8-26$ & $22.6 \pm 2.26$ & $19.4 \pm 1.28$ & $20.6 \pm 1.36$ & $22.4 \pm 1.59$ & $19.8 \pm 1.05$ \\
$\mathbf{N a}(\mathbf{m g} / \mathbf{L})$ & $7.5-13.4$ & $10.6 \pm 1.23$ & $9.4 \pm 0.66$ & $8.8 \pm 0.47$ & $10.0 \pm 0.72$ & $8.9 \pm 0.52$ \\
$\mathbf{K}(\mathbf{m g} / \mathbf{L})$ & $2.6-5.3$ & $4.4 \pm 0.70$ & $3.9 \pm 0.52$ & $3.9 \pm 0.45$ & $4.4 \pm 0.49$ & $3.6 \pm 0.42$ \\
$\mathbf{N O}_{3}-\mathbf{N}(\mathbf{\mu g} / \mathbf{L})$ & $220-557$ & $390.3 \pm 42.6$ & $351.3 \pm 45.7$ & $261.4 \pm 25.1$ & $338.4 \pm 57.4$ & $279.3 \pm 23.5$ \\
$\mathbf{P}(\mathbf{\mu g} / \mathbf{L})$ & $110-290$ & $238.17 \pm 20.36$ & $197.96 \pm 20.68$ & $150.67 \pm 13.70$ & $150.67 \pm 18.46$ & $143.42 \pm 12.66$ \\
\hline
\end{tabular}

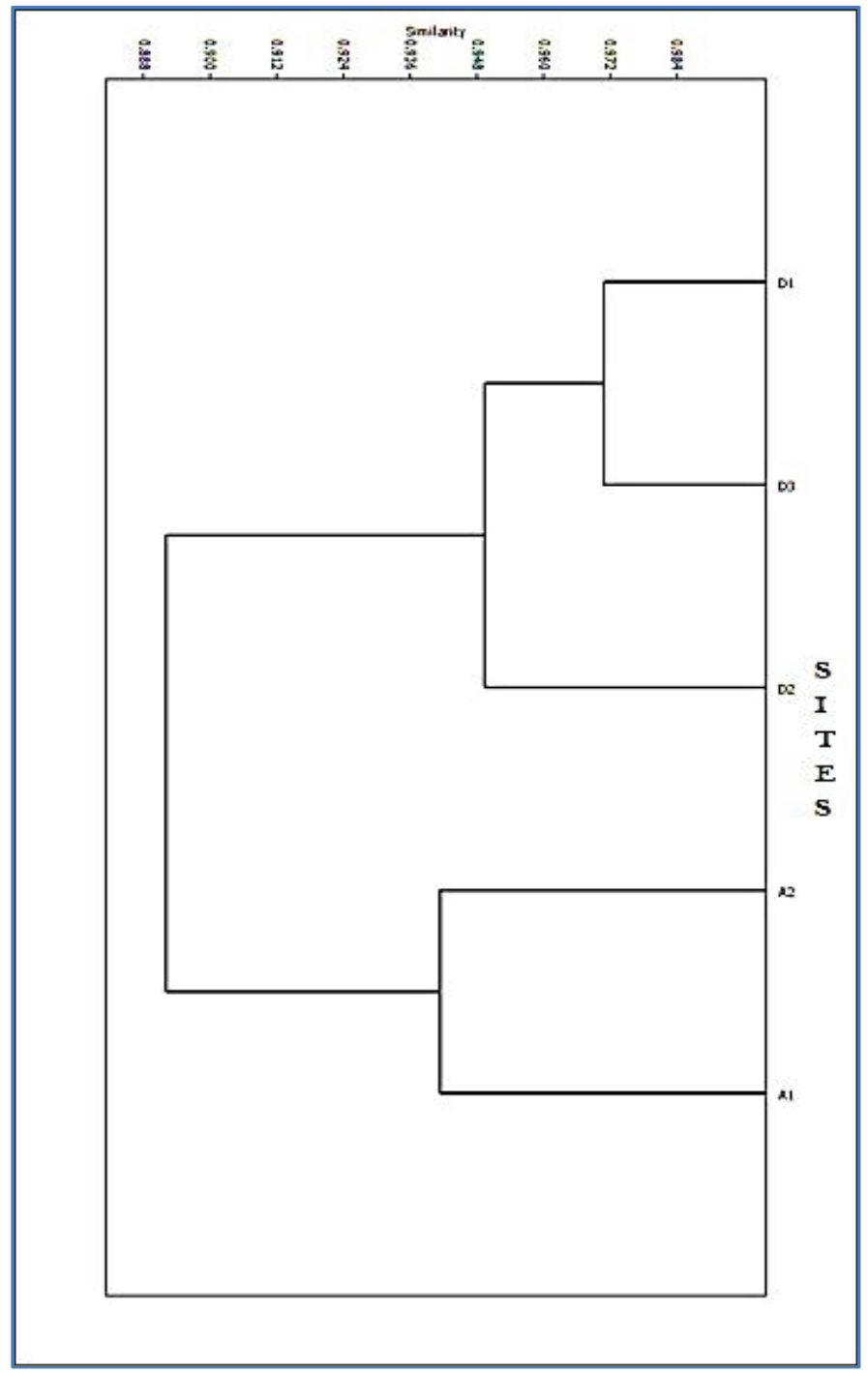

Figure 2. Dendrogram (Similarity Index) derived from hierarchical cluster analysis of water variables. 
Table 2. Significance levels of water variables at 0.01 and 0.05 (2-tailed) correlations

\begin{tabular}{|c|c|c|c|c|c|c|c|c|c|c|c|}
\hline & & $\mathrm{T}$ & $\mathrm{pH}$ & Conductivity & Bicarbonates & $\mathrm{Cl}$ & $\mathrm{Ca}$ & $\mathrm{Mg}$ & $\mathrm{Na}$ & $\mathrm{K}$ & Nitrate-N $\mathrm{F}$ \\
\hline $\mathrm{T}$ & $\begin{array}{l}\text { Pearson Correlation } \\
\text { Sig. (2-tailed) }\end{array}$ & 1 & & & & & & & & & \\
\hline $\mathrm{pH}$ & $\begin{array}{c}\text { Pearson Correlation } \\
\text { Sig. (2-tailed) } \\
\end{array}$ & $\begin{array}{l}.918^{* *} \\
.000 \\
\end{array}$ & 1 & & & & & & & & \\
\hline Conductivity & $\begin{array}{c}\text { Pearson Correlation } \\
\text { Sig. (2-tailed) } \\
\end{array}$ & $\begin{array}{c}-.917^{* *} \\
.000 \\
\end{array}$ & $\begin{array}{l}-.932^{* *} \\
.000 \\
\end{array}$ & 1 & & & & & & & \\
\hline Bicarbonates & $\begin{array}{c}\text { Pearson Correlation } \\
\text { Sig. (2-tailed) }\end{array}$ & $\begin{array}{c}-.736^{* *} \\
.000 \\
\end{array}$ & $\begin{array}{r}-.507^{*} \\
.012 \\
\end{array}$ & $\begin{array}{l}.585^{* *} \\
.003 \\
\end{array}$ & 1 & & & & & & \\
\hline $\mathrm{Cl}$ & $\begin{array}{c}\text { Pearson Correlation } \\
\text { Sig. (2-tailed) } \\
\end{array}$ & $\begin{array}{l}.266 \\
.209 \\
\end{array}$ & $\begin{array}{l}.159 \\
.459 \\
\end{array}$ & $\begin{array}{l}-.308 \\
.143 \\
\end{array}$ & $\begin{array}{l}-.228 \\
.285 \\
\end{array}$ & 1 & & & & & \\
\hline $\mathrm{Ca}$ & $\begin{array}{c}\text { Pearson Correlation } \\
\text { Sig. (2-tailed) }\end{array}$ & $\begin{array}{c}.531^{* *} \\
.008 \\
\end{array}$ & $\begin{array}{c}.568^{* *} \\
.004 \\
\end{array}$ & $\begin{array}{l}.617^{* *} \\
.001 \\
\end{array}$ & $\begin{array}{l}.222 \\
.296 \\
\end{array}$ & $\begin{array}{l}.114 \\
.595 \\
\end{array}$ & 1 & & & & \\
\hline $\mathrm{Mg}$ & $\begin{array}{c}\text { Pearson Correlation } \\
\text { Sig. (2-tailed) }\end{array}$ & $\begin{array}{c}.803^{* *} \\
.000 \\
\end{array}$ & $\begin{array}{l}.715^{* *} \\
.000 \\
\end{array}$ & $\begin{array}{l}.800^{* *} \\
.000 \\
\end{array}$ & $\begin{array}{l}.613^{* *} \\
.001 \\
\end{array}$ & $\begin{array}{r}.307 \\
.144 \\
\end{array}$ & $\begin{array}{l}.329 \\
.116 \\
\end{array}$ & 1 & & & \\
\hline $\mathrm{Na}$ & $\begin{array}{c}\text { Pearson Correlation } \\
\text { Sig. (2-tailed) } \\
\end{array}$ & $\begin{array}{c}.641^{* *} \\
.001 \\
\end{array}$ & $\begin{array}{c}.672^{* *} \\
.000 \\
\end{array}$ & $\begin{array}{l}.641^{* *} \\
.001 \\
\end{array}$ & $\begin{array}{l}.299 \\
.156 \\
\end{array}$ & $\begin{array}{l}.173 \\
.420 \\
\end{array}$ & $\begin{array}{l}.532^{* *} \\
.007 \\
\end{array}$ & $\begin{array}{l}.590^{* *} \\
.002 \\
\end{array}$ & 1 & & \\
\hline K & $\begin{array}{l}\text { Pearson Correlation } \\
\text { Sig. (2-tailed) }\end{array}$ & $\begin{array}{c}.661^{* *} \\
.000 \\
\end{array}$ & $\begin{array}{c}-.726^{* *} \\
.000 \\
\end{array}$ & $\begin{array}{l}.709^{* *} \\
.000\end{array}$ & $\begin{array}{l}.249 \\
.240 \\
\end{array}$ & $\begin{array}{l}.258 \\
.223 \\
\end{array}$ & $\begin{array}{c}.677^{* *} \\
.000 \\
\end{array}$ & $\begin{array}{l}.497^{*} \\
.014 \\
\end{array}$ & $\begin{array}{l}.813^{* *} \\
.000 \\
\end{array}$ & 1 & \\
\hline Nitrate- N & $\begin{array}{c}\text { Pearson Correlation } \\
\text { Sig. (2-tailed) }\end{array}$ & $\begin{array}{l}.356 \\
.088 \\
\end{array}$ & $\begin{array}{l}.382 \\
.065 \\
\end{array}$ & $\begin{array}{l}-.312 \\
.138 \\
\end{array}$ & $\begin{array}{c}-.408^{*} \\
.048 \\
\end{array}$ & $\begin{array}{l}.295 \\
.162 \\
\end{array}$ & $\begin{array}{l}.210 \\
.324 \\
\end{array}$ & $\begin{array}{c}.124 \\
.563 \\
\end{array}$ & $\begin{array}{l}.083 \\
.700 \\
\end{array}$ & $\begin{array}{l}.073 \\
.736 \\
\end{array}$ & 1 \\
\hline$P$ & $\begin{array}{l}\text { Pearson Correlation } \\
\text { Sig. (2-tailed) }\end{array}$ & $\begin{array}{r}-.485^{*} \\
.016\end{array}$ & $\begin{array}{c}-.530^{* *} \\
.008\end{array}$ & $\begin{array}{l}.531^{* *} \\
.008\end{array}$ & $\begin{array}{l}.187 \\
.381\end{array}$ & $\begin{array}{l}.343 \\
.101\end{array}$ & $\begin{array}{l}.614^{* *} \\
.001\end{array}$ & $\begin{array}{l}.414^{*} \\
.044\end{array}$ & $\begin{array}{l}.745^{* *} \\
.000\end{array}$ & $\begin{array}{l}.784^{* *} \\
.000\end{array}$ & $\begin{array}{l}.378 \\
.069\end{array}$ \\
\hline
\end{tabular}

** Correlation is significant at 0.01 level (2-tailed)

* Correlation is significant at 0.05 level (2-tailed)

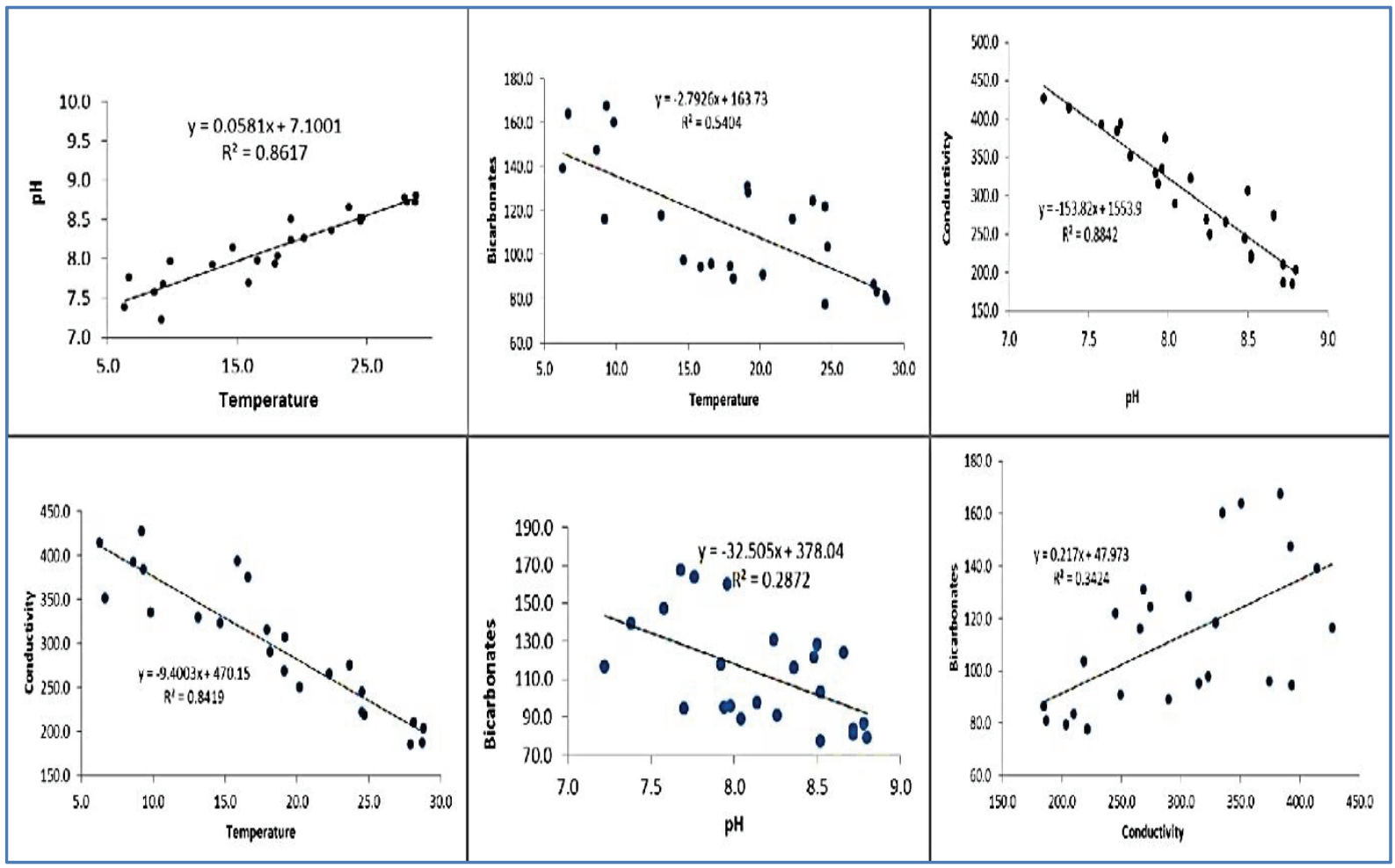

Figure 3. Line of best-fit $\left(\mathrm{R}^{2}\right)$ describing intra-aqueous interdependence of water variables. 


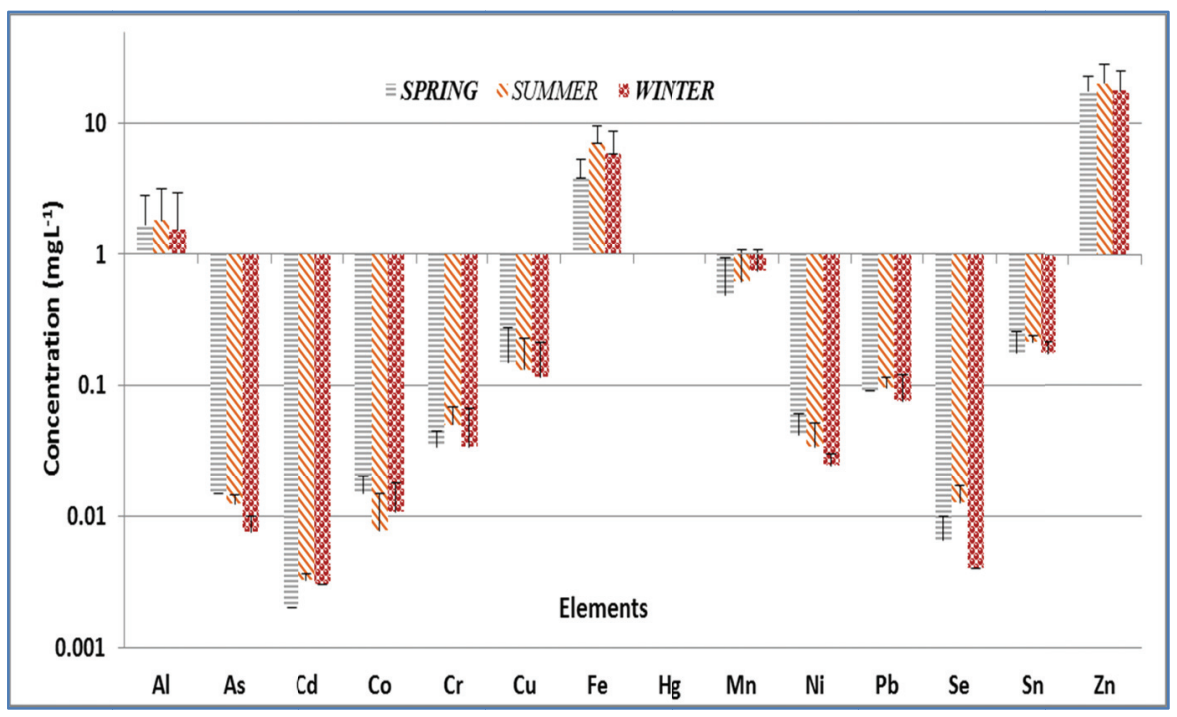

Figure 4. Average trace-metal concentrations and seasonal variation in micro and trace ellements in lake waters

Table 3. Significance levels of trace elements in epilimnion at 0.01 and 0.05 (2-tailed) correlations

\begin{tabular}{|c|c|c|c|c|c|c|c|c|c|c|c|c|c|c|c|}
\hline & & $\mathrm{Al}$ & $\mathrm{As}$ & $\mathrm{Cd}$ & $\mathrm{Co}$ & $\mathrm{Cr}$ & $\mathrm{Cu}$ & $\mathrm{Fe}$ & $\mathrm{Hg}$ & $\mathrm{Mn}$ & $\mathrm{Ni}$ & $\mathrm{Pb}$ & $\mathrm{Se}$ & $\mathrm{Sn}$ & $\mathrm{Zn}$ \\
\hline $\mathrm{Al}$ & $\begin{array}{c}\text { Pearson Correlation } \\
\text { Sig. (2-tailed) }\end{array}$ & 1 & & & & & & & & & & & & & \\
\hline As & $\begin{array}{c}\text { Pearson Correlation } \\
\text { Sig. (2-tailed) }\end{array}$ & $\begin{array}{l}.350 \\
.201\end{array}$ & 1 & & & & & & & & & & & & \\
\hline $\mathrm{Cd}$ & $\begin{array}{c}\text { Pearson Correlation } \\
\text { Sig. (2-tailed) }\end{array}$ & $\begin{array}{l}.201 \\
.152 \\
.589 \\
\end{array}$ & $\begin{array}{l}.898^{* *} \\
.000 \\
\end{array}$ & 1 & & & & & & & & & & & \\
\hline Co & $\begin{array}{c}\text { Pearson Correlation } \\
\text { Sig. (2-tailed) }\end{array}$ & $\begin{array}{l}.652^{* *} \\
.008 \\
\end{array}$ & $\begin{array}{l}.488 \\
.065 \\
\end{array}$ & $\begin{array}{l}.285 \\
.303 \\
\end{array}$ & 1 & & & & & & & & & & \\
\hline $\mathrm{Cr}$ & $\begin{array}{c}\text { Pearson Correlation } \\
\text { Sig. (2-tailed) }\end{array}$ & $\begin{array}{l}.863^{* *} \\
.000 \\
\end{array}$ & $\begin{array}{l}.325 \\
.237 \\
\end{array}$ & $\begin{array}{l}.200 \\
.475 \\
\end{array}$ & $\begin{array}{l}.412 \\
.127 \\
\end{array}$ & 1 & & & & & & & & & \\
\hline $\mathrm{Cu}$ & $\begin{array}{c}\text { Pearson Correlation } \\
\text { Sig. (2-tailed) }\end{array}$ & $\begin{array}{l}.870^{* *} \\
.000 \\
\end{array}$ & $\begin{array}{l}.448 \\
.094 \\
\end{array}$ & $\begin{array}{l}.227 \\
.416 \\
\end{array}$ & $\begin{array}{l}.769^{* *} \\
.001 \\
\end{array}$ & $\begin{array}{l}.766^{* *} \\
.001 \\
\end{array}$ & 1 & & & & & & & & \\
\hline $\mathrm{Fe}$ & $\begin{array}{c}\text { Pearson Correlation } \\
\text { Sig. (2-tailed) }\end{array}$ & $\begin{array}{l}-.213 \\
.447 \\
\end{array}$ & $\begin{array}{l}.399 \\
.141 \\
\end{array}$ & $\begin{array}{l}.610^{*} \\
.016 \\
\end{array}$ & $\begin{array}{l}.015 \\
.959 \\
\end{array}$ & $\begin{array}{l}-.134 \\
.635 \\
\end{array}$ & $\begin{array}{r}.047 \\
.868 \\
\end{array}$ & 1 & & & & & & & \\
\hline $\mathrm{Hg}$ & & ${ }^{\mathrm{a}}$ &. $\mathrm{a}$ &.${ }^{\mathrm{a}}$ &.${ }^{\mathrm{a}}$ &.$^{\mathrm{a}}$ &.$^{\mathrm{a}}$ &.$^{\mathrm{a}}$ &.$^{\mathrm{a}}$ & & & & & & \\
\hline Mn & $\begin{array}{c}\text { Pearson Correlation } \\
\text { Sig. (2-tailed) }\end{array}$ & $\begin{array}{l}.323 \\
.241 \\
\end{array}$ & $\begin{array}{c}-.135 \\
.632 \\
\end{array}$ & $\begin{array}{c}-.176 \\
.531 \\
\end{array}$ & $\begin{array}{l}.163 \\
.561 \\
\end{array}$ & $\begin{array}{l}.339 \\
.216 \\
\end{array}$ & $\begin{array}{l}.139 \\
.621 \\
\end{array}$ & $\begin{array}{r}-.127 \\
.652 \\
\end{array}$ & . & 1 & & & & & \\
\hline $\mathrm{Ni}$ & $\begin{array}{c}\text { Pearson Correlation } \\
\text { Sig. (2-tailed) }\end{array}$ & $\begin{array}{l}.543^{*} \\
.036 \\
\end{array}$ & $\begin{array}{l}.661^{\text {** }} \\
.007\end{array}$ & $\begin{array}{l}.485 \\
.067\end{array}$ & $\begin{array}{l}.626^{*} \\
.013 \\
\end{array}$ & $\begin{array}{l}.455 \\
.089 \\
\end{array}$ & $\begin{array}{l}.520^{*} \\
.047 \\
\end{array}$ & $\begin{array}{l}.019 \\
.945 \\
\end{array}$ & . & $\begin{array}{l}.289 \\
.297\end{array}$ & 1 & & & & \\
\hline $\mathrm{Pb}$ & $\begin{array}{c}\text { Pearson Correlation } \\
\text { Sig. (2-tailed) }\end{array}$ & $\begin{array}{l}.115 \\
.682 \\
\end{array}$ & $\begin{array}{l}.492 \\
.062 \\
\end{array}$ & $\begin{array}{l}.671^{* *} \\
.006 \\
\end{array}$ & $\begin{array}{c}-.019 \\
.945 \\
\end{array}$ & $\begin{array}{l}.292 \\
.292 \\
\end{array}$ & $\begin{array}{l}.203 \\
.468 \\
\end{array}$ & $\begin{array}{l}.555^{*} \\
.032 \\
\end{array}$ & . & $\begin{array}{r}.059 \\
.834 \\
\end{array}$ & $\begin{array}{l}.264 \\
.341 \\
\end{array}$ & 1 & & & \\
\hline $\mathrm{Se}$ & $\begin{array}{c}\text { Pearson Correlation } \\
\text { Sig. (2-tailed) }\end{array}$ & $\begin{array}{c}-.180 \\
.521 \\
\end{array}$ & $\begin{array}{l}.417 \\
.122 \\
\end{array}$ & $\begin{array}{l}.473 \\
.075 \\
\end{array}$ & $\begin{array}{c}-.353 \\
.197 \\
\end{array}$ & $\begin{array}{l}.034 \\
.905 \\
\end{array}$ & $\begin{array}{l}.124 \\
.659 \\
\end{array}$ & $\begin{array}{l}.245 \\
.380 \\
\end{array}$ & . & $\begin{array}{r}-.427 \\
.112 \\
\end{array}$ & $\begin{array}{l}-.074 \\
.795 \\
\end{array}$ & $\begin{array}{l}.373 \\
.171 \\
\end{array}$ & 1 & & \\
\hline Sn & $\begin{array}{c}\text { Pearson Correlation } \\
\text { Sig. (2-tailed) }\end{array}$ & $\begin{array}{l}.276 \\
.319 \\
\end{array}$ & $\begin{array}{l}.409 \\
.130 \\
\end{array}$ & $\begin{array}{l}.510 \\
.052 \\
\end{array}$ & $\begin{array}{l}.166 \\
.553 \\
\end{array}$ & $\begin{array}{l}.457 \\
.087 \\
\end{array}$ & $\begin{array}{l}.219 \\
.432 \\
\end{array}$ & $\begin{array}{l}.491 \\
.063 \\
\end{array}$ & 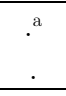 & $\begin{array}{l}.399 \\
.140 \\
\end{array}$ & $\begin{array}{l}.502 \\
.057 \\
\end{array}$ & $\begin{array}{l}.433 \\
.107 \\
\end{array}$ & $\begin{array}{l}.073 \\
.795 \\
\end{array}$ & 1 & \\
\hline $\mathrm{Zn}$ & $\begin{array}{c}\text { Pearson Correlation } \\
\text { Sig. (2-tailed) }\end{array}$ & $\begin{array}{l}.088 \\
.755 \\
\end{array}$ & $\begin{array}{l}.099 \\
.725 \\
\end{array}$ & $\begin{array}{l}.291 \\
.292 \\
\end{array}$ & $\begin{array}{c}-.125 \\
.658 \\
\end{array}$ & $\begin{array}{l}.167 \\
.552 \\
\end{array}$ & $\begin{array}{l}.043 \\
.880 \\
\end{array}$ & $\begin{array}{l}.224 \\
.422 \\
\end{array}$ & . & $\begin{array}{l}.209 \\
.455 \\
\end{array}$ & $\begin{array}{l}.315 \\
.253 \\
\end{array}$ & $\begin{array}{l}.591^{*} \\
.020\end{array}$ & $\begin{array}{l}.149 \\
.597\end{array}$ & $\begin{array}{l}.283 \\
.306\end{array}$ & 1 \\
\hline
\end{tabular}

** Correlation is significant at 0.01 level (2-tailed)

a Cannot be computed because at least one of the variables is constant (BDL)

* Correlation is significant at 0.05 level (2-tailed) 


\subsection{Surface Sediments}

The observed range of H-ion concentration in surface sediments is 6.16 to 8.35 and the respective mean site values include $7.22 \pm 0.60,7.30 \pm 0.64,7.58 \pm 0.42,7.20 \pm 0.58,7.49 \pm 0.56$. The sediment $\mathrm{pH}$ generally remained on the basic side but slight acidic nature is noticed during late summer probably due to temperature determined decomposition of organic matter releasing acidic by-products. Alkaline $\mathrm{pH}$ records of the sediment in general determine the catchment calcite predominance whereas slight summer diminution is temperature improved OM decomposition effect. Sediment conductivity ranged between 234 and $498 \mathrm{\mu S} / \mathrm{cm}$ at $25{ }^{\circ} \mathrm{C}$ and the average inter-site comparison is as $395.4 \pm 55.7,312.6 \pm 47.2$, $293.3 \pm 36.4,363.3 \pm 50.9$ and $323.3 \pm 38.4$ respectively. The sediment bicarbonate content has minima of 116 and maxima of $264 \mathrm{mg} / \mathrm{Kg}$ and varied between the sites as $187.4 \pm 25.3,193.5 \pm 37.2,169.8 \pm 41.5$, $172.6 \pm 41.9$ and $189.6 \pm 42.3$. Similarly, ranges and average site percentage dry-weight variations in sediment organic $\mathrm{C}$, organic matter, total $\mathrm{N}$ and $\mathrm{C} / \mathrm{N}$ ratio are summarized in Table 4 . A temporal trend of the above seven sediment characteristics for contiguous eight seasons of two year study can be visualised as inconsequential whereas $\mathrm{Ca}-\mathrm{Si}$ dominance is depicted throughout. Table 5 depicts significant correlation for conductivity with organic $\mathrm{C}$ and organic matter as the latter serves an established source for nutrient ions. Similar is the case with sediment organic C, organic matter and total $\mathrm{N}$ as being complementary to one another. The typical $<10 \mathrm{C} / \mathrm{N}$ implies autochthonous $\mathrm{OM}$, besides low decomposition rates controlled by $\mathrm{N}$ budgeting and speciation via biochemical (enzyme and $\mathrm{pH}$ ) fluctuations under alkaline conditions. Higher temperature and lower N:P ratio during summer develop the $\mathrm{P}$ internal loading process. This $\mathrm{P}$ recycling mediates via degradation (oxic), denitrification and sulphate reduction. Nonetheless, higher $\mathrm{Al}, \mathrm{Ca}$ and $\mathrm{Fe}$ proportions in sediments inactivate $\mathrm{P}$ mobilization. The estimated percentage elemental composition (Fig. 5) of surface sediments on dry weight basis revealed the order $\mathrm{Si}>\mathrm{Ca}>\mathrm{Mg}>\mathrm{K}>\mathrm{Na}>\mathrm{P}>\mathrm{S}>\mathrm{Cl}$. Besides, the micro and trace element quantification (Fig.5) represent the descending series of $\mathrm{Fe}>\mathrm{Al}>\mathrm{Zn}>\mathrm{Mn}>\mathrm{Cu}>\mathrm{Cr}>\mathrm{Ni}>\mathrm{Co}>\mathrm{As}>\mathrm{Sn}>$ $\mathrm{Pb}>\mathrm{Cd}$ while $\mathrm{Hg}$ and Se remained below detection level (BDL).

Table 4. Ranges and average site percentage dry-weights of sediment variables

\begin{tabular}{ccccccc}
\hline SEDIMENT-VARIABLE & RANGE & A1 & A2 & D1 & D2 & D3 \\
\hline ORGANIC-CARBON (\%) & 6.7 to 15.21 & $11.05 \pm 2.38$ & $9.42 \pm 1.58$ & $8.67 \pm 0.84$ & $9.83 \pm 1.27$ & $7.85 \pm 0.77$ \\
ORGANIC-MATTER (\%) & 11.5 to 26.22 & $19.035 \pm 4.096$ & $16.238 \pm 2.726$ & $14.940 \pm 1.442$ & $16.939 \pm 2.178$ & $13.526 \pm 1.327$ \\
TOTAL-NITROGEN (\%) & 1.09 to 2.87 & $1.948 \pm 0.332$ & $1.828 \pm 0.322$ & $1.864 \pm 0.306$ & $1.900 \pm 0.174$ & $2.458 \pm 0.322$ \\
C/N RATIO & 2.55 to 7.53 & $5.710 \pm 1.018$ & $5.208 \pm 0.720$ & $4.789 \pm 0.948$ & $5.175 \pm 0.554$ & $3.229 \pm 0.373$ \\
\hline
\end{tabular}

Table 5. Significance levels of sediment variables at 0.01 and 0.05 (2-tailed) correlations

\begin{tabular}{|c|c|c|c|c|c|c|c|c|}
\hline & & $\mathrm{pH}$ & Conductivity & Bicarbonate & $\mathrm{OC}$ & OM & TN & $\mathrm{C} / \mathrm{N}$ ratio \\
\hline $\mathrm{pH}$ & $\begin{array}{c}\text { Pearson Correlation } \\
\text { Sig. (2-tailed) } \\
\end{array}$ & 1 & & & & & & \\
\hline Conductivity & $\begin{array}{l}\text { Pearson Correlation } \\
\text { Sig. (2-tailed) }\end{array}$ & $\begin{array}{l}-.350 \\
.395\end{array}$ & 1 & & & & & \\
\hline Bicarbonate & $\begin{array}{l}\text { Pearson Correlation } \\
\text { Sig. (2-tailed) }\end{array}$ & $\begin{array}{l}.720^{*} \\
.044 \\
\end{array}$ & $\begin{array}{c}-.039 \\
.928 \\
\end{array}$ & 1 & & & & \\
\hline $\mathrm{OC}$ & $\begin{array}{l}\text { Pearson Correlation } \\
\text { Sig. (2-tailed) } \\
\end{array}$ & $\begin{array}{l}.086 \\
.840 \\
\end{array}$ & $\begin{array}{l}.853^{* *} \\
.007 \\
\end{array}$ & $\begin{array}{l}.357 \\
.385 \\
\end{array}$ & 1 & & & \\
\hline OM & $\begin{array}{c}\text { Pearson Correlation } \\
\text { Sig. (2-tailed) } \\
\end{array}$ & $\begin{array}{l}.086 \\
.840 \\
\end{array}$ & $\begin{array}{l}.854^{* *} \\
.007\end{array}$ & $\begin{array}{l}.356 \\
.386 \\
\end{array}$ & $\begin{array}{c}1.000^{* *} \\
.000\end{array}$ & 1 & & \\
\hline $\mathrm{TN}$ & $\begin{array}{l}\text { Pearson Correlation } \\
\text { Sig. (2-tailed) } \\
\end{array}$ & $\begin{array}{l}.320 \\
.439 \\
\end{array}$ & $\begin{array}{l}.678 \\
.065 \\
\end{array}$ & $\begin{array}{l}.504 \\
.203 \\
\end{array}$ & $\begin{array}{l}.860^{* *} \\
.006 \\
\end{array}$ & $\begin{array}{l}.860^{* *} \\
.006\end{array}$ & 1 & \\
\hline $\mathrm{C} / \mathrm{N}$ ratio & $\begin{array}{l}\text { Pearson Correlation } \\
\text { Sig. (2-tailed) } \\
\end{array}$ & $\begin{array}{c}-.545 \\
.163 \\
\end{array}$ & $\begin{array}{l}.279 \\
.503 \\
\end{array}$ & $\begin{array}{r}-.275 \\
.510 \\
\end{array}$ & $\begin{array}{l}.130 \\
.759 \\
\end{array}$ & $\begin{array}{l}.129 \\
.760 \\
\end{array}$ & $\begin{array}{l}-.352 \\
.392 \\
\end{array}$ & 1 \\
\hline
\end{tabular}

* Correlation is significant at 0.05 level (2-tailed)

** Correlation is significant at 0.01 level (2-tailed) 


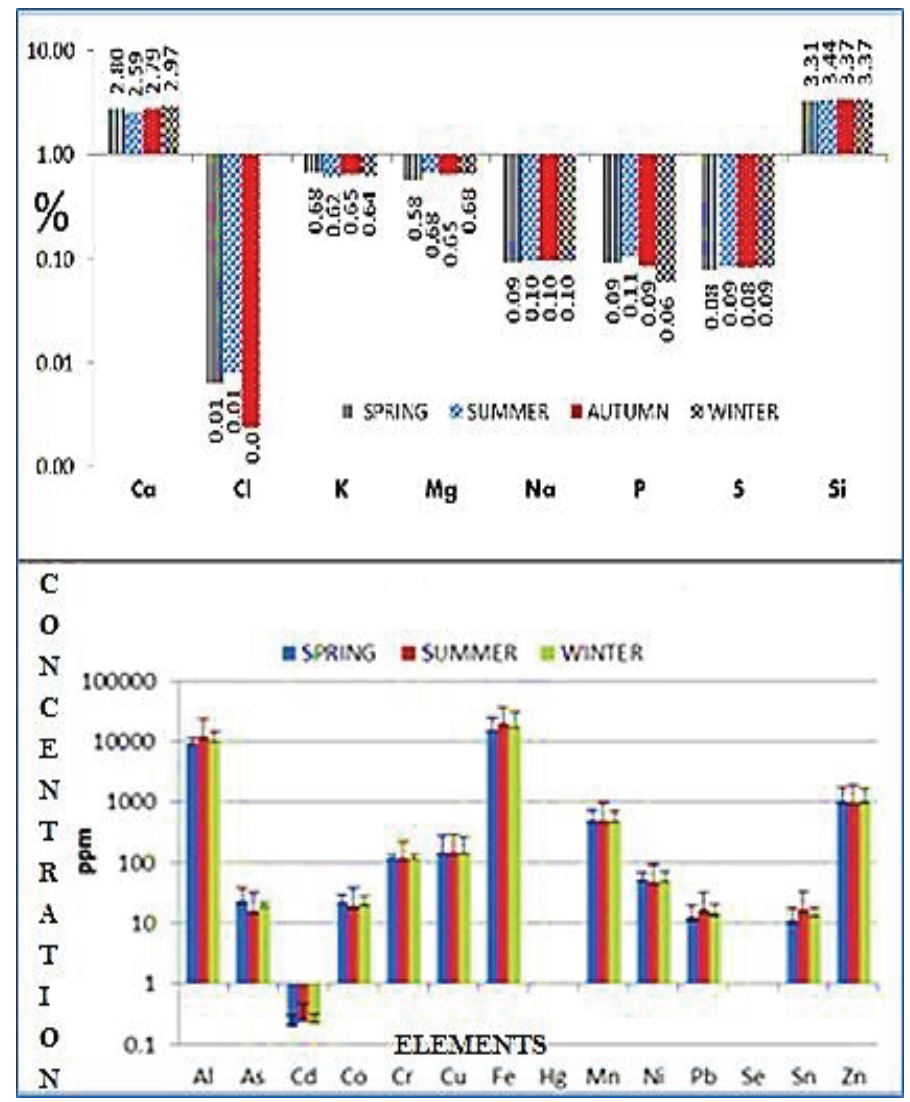

Figure 5. Mean percentage elemental composition of surface sediments.

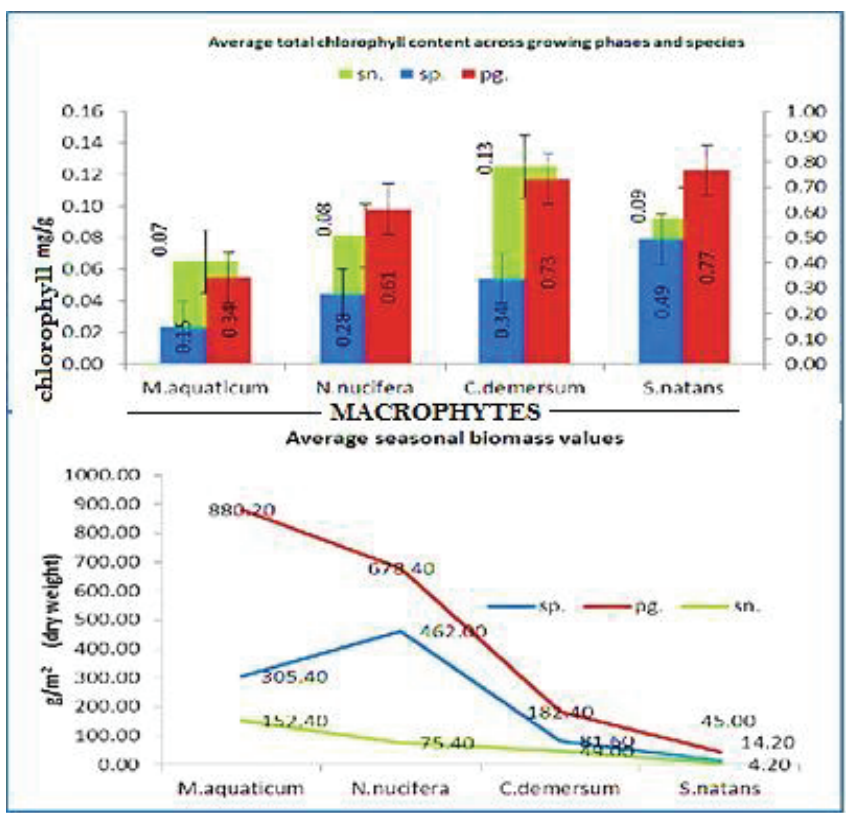

Figure 6. Total chlorophyll and dry-weight biomass of the experimental species. 


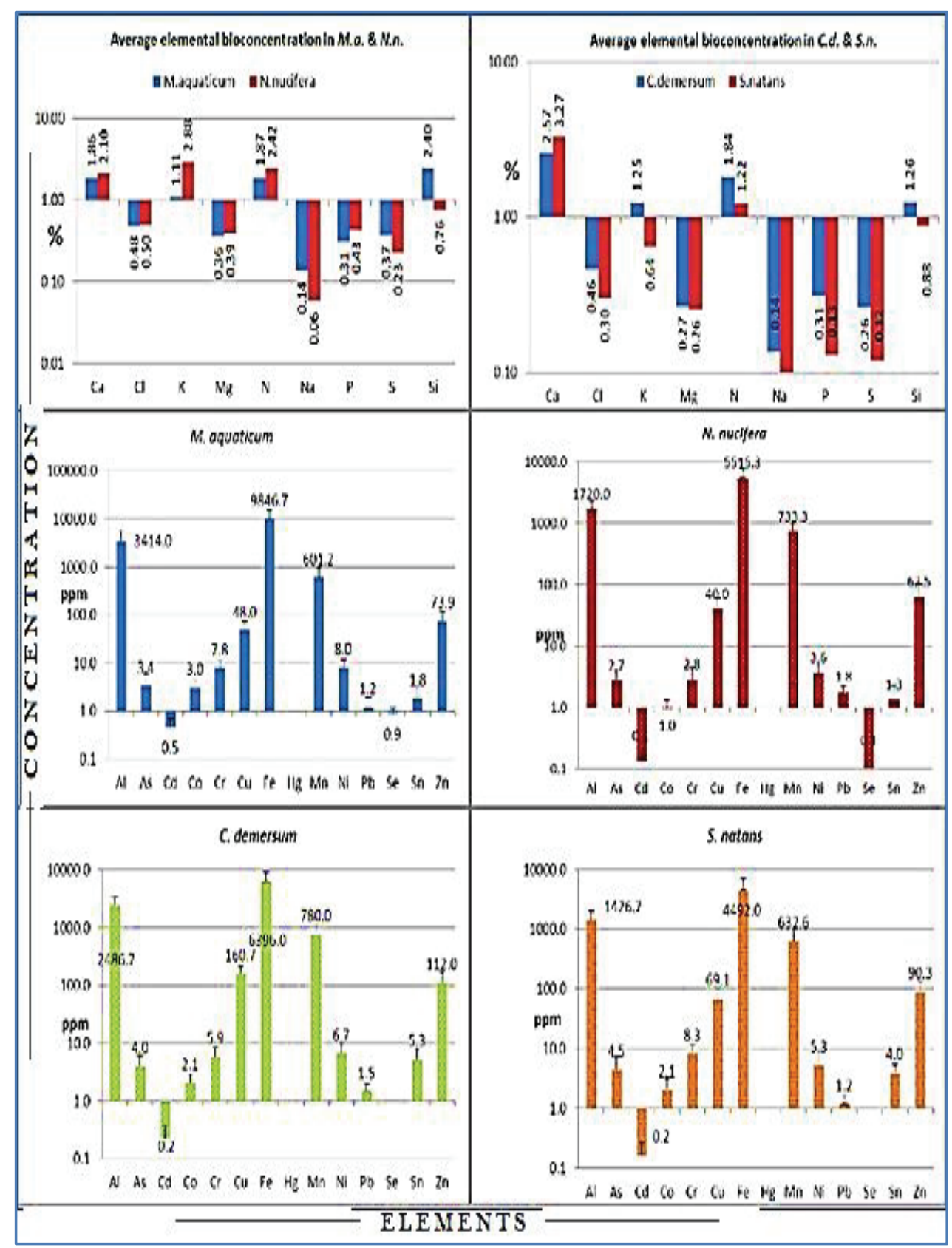

Figure 7. Bioaccumulation profile of nutrients and trace elements.

\subsection{Macrophytes}

The productive capacity of the investigated macrophytes calculated on the basis of total chlorophyll content and dry-weight biomass for each experimental species in the three major growth phases is presented in Fig.6. Further, the tissue concentration of nutrients and trace elements illustrated in Fig.7 differ species wise but well correlate with ambient water and sediment media. M. aquaticum accumulated $49 \%$ of the dry weight biomass annually whereas for $N$. nucifera amounted to $38 \%, C$. demersum to $10 \%$ and S. natans contributed only $3 \%$. The analysis of four dominant ubiquitous life forms of macrophytes signified marked variance in productivity pattern and specific growth rates of the order emergent $M$. aquaticum $>$ rooted floating $N$. nucifera $>$ submerged $C$. demersum $>$ free floating $S$. natans well in agreement to their physiognomy and micro-habitat. 


\section{Discussion}

The limnochemical statistics that evolved during the study retro expound definite and obvious development of physicochemical and biological configuration of the select sites in both lakes under multiple anthropogenic stress [38]. The chemical composition of water closely related to dual factors of concerned catchment and human intervention thereof [39]. The lakes studied deem to be classified under eutrophic category with considerable differences in bio-physicochemical milieu due to varied nature of operative anthropogenic stress [28]. But for the semi-drainage type hydrology flushing via regular outflow channels supplemented by ephemeral and sub-aqueous inputs is vital for their self-reclaimed homeostasis since the basin holding-time of water keeps low (2/2 months). A higher $\mathrm{pH}$ associated with elevated temperature and alkaline conditions is reported to favour co-precipitation of carbonate and phosphate due to rapid carbon assimilation from dissolved bicarbonates [26] and [40]. Despite a temporary inverse relationship of bicarbonate concentration and $\mathrm{pH}$ during peak-growth summer days, the enriched alkaline $\mathrm{pH}$ switch ascribes to equivalent carbonate dominance [1] and [27]. Conductivity describes the trophic status in terms of total nutritive ionic strength of the water column. Its decline in peak growth season implies ionic depletion by photoautotrophic consumption and biogenic calcification (precipitation of $\mathrm{CaCO}_{3}$ ) commonly referred to as seasonal whiting or clouding of lakes [41]. The ionic strength (conductivity) measures vindicate superior eutrophy (solute richness) besides autotrophic assimilation and biocalcification contributing towards its summer falls [28] and [42]. The lime grade catchment characteristic contributed to Ca dominance phenomenon and hard water type classification [33] and [43]. $\mathrm{K}$ features base association with agricultural runoff and $\mathrm{Cl}$ to faunal organic pollution [44] and [45]. A range of human actions involving agriculture, farming, sewage, factories, etc. result in $\mathrm{N}$ species inflow [46]. The spring fed basins of both the lakes and preferential reduced $\mathrm{NH}_{4}{ }^{+}$autotrophic assimilation instead of oxidised $\mathrm{NO}_{3}{ }^{-}$also greater concentration of nitrate $-\mathrm{N}$ in water [29] and [47]. Additionally, isolated geogenic $\mathrm{N}$ pockets are ascribed as evaporative deposits in phyllite, schist and carbonate bed rocks in Kashmir [33]. Forest surface runoff too contain $\leqslant 20 \mathrm{mgL}^{-1}$ nitrate as a natural source to lakes [48]. Summer decreases of the least abundant macronutrient (P) limits bio-productivity owing to its utilization and carbonate coprecipitation [49]. The amount of $\mathrm{N}$ and $\mathrm{P}$ forms is intimately related to biological productivity of aquatic ecosystems [47]. Higher temperatures favour bio-production and thereby elevate $\mathrm{pH}$ significantly. So, the factors of temperature and $\mathrm{pH}$ clearly state that improved growth environment for biological uptake and calcite co-precipitation of ions reduce their epilimnion concentrations in peak growth summer cycle. Such a systematics attributed to photosynthetically induced precipitation and utilization by autotrophs is concomitant to latest studies [50]. Anthropogenic effluents are established primary sources of traces in surface waters [30] and [51]. Pertinently, such proportions exceed maximum permissible limits for drinking water but are suitable as irrigation liquid ordinarily [31], [32] and [52]. The complexing phenomena of heavy metals with carbonate and BIC limit their direct or indirect repercussions [53]. Now, the possible source identification includes solid and liquid discards from an array of utilities namely alloys, storage batteries, pesticides, wood preservatives, electroplating, pigments, fertilizers, porcelain, glass, electrical wiring, roofing, utensils, piping, chemicals, paints, vapour lamps, mirror coatings, thermometers, catalysts, ceramics, fossil fuels, electronics, solder and so on [17].

The abiotic quiescent zoneof sediments is suitable long-term indicator of lake environmental conditions [54] and [55]. Sediment nutrient constitution corresponds to catchment land use [56]. They provide settling space for water carried elements, both acquired and innate, in the form of ions, organic/inorganic- complexes and dissolved/suspended- matter associations [57]. Sediments reflect weaker source but stronger sink behaviour and capacity depicted via transformations of adsorption/desorption, mineralization/demineralization, bioassimilation and burial phenomenon [58]. Sediments' $\mathrm{pH}$ can be labelled as the principal factor enacting nutrient accessibility and movement [59]. The seasonal organic $\mathrm{C}$ changes in sediments aptly describe a pattern of temperature induced decrement during summers fulfilling the mineralization promotion [33] and [60]. The analysis of superficial sediment chemical characters reveals connections with trophic index [61]. During the course of the study most of the nutrients could be observed to follow similar tendency both in surface sediments and overlying water column ominous of systematic interchange [62]. A general gradual decline in their concentration till culmination of active macrophytic growth phase can be associated with active/passive bioaccumulation 
or anoxic release from sediments [63]. Its possible reason could be $\mathrm{NH}_{4}{ }^{+}$microbial preference instead of $\mathrm{NO}_{3}{ }^{-}$stimulating decomposition despite eutrophied state [34] and [64]. Improved $\mathrm{N}: \mathrm{P}$ ratios parallel chronic nitrogen influx in P-limited lakes [65], however, N-limited lakes suffer greater eutrophication catastrophes [66]. Curbing the external $\mathrm{N}$ and $\mathrm{P}$ loads is effective in remediation but the sediment internal loading supplement compensates the loss [35] and [67]. Meanwhile sediment dredging is beneficial for internal nutrient deloading only after the external inputs are checked [68]. Trace metals bound to $\mathrm{OM}$ or $\mathrm{Fe} / \mathrm{Mn}$ - oxides separate on their decomposition and reductive dissolution respectively near hypolimnion-sediment overlap [35], [36] and [69]. The lower [Ca: Al] values suggest exsitu human cause for PTE transport to the designated lacustrine sites [58]. Nonetheless, the mobility of PTE's is effectively curtailed by OM enriched sediments and calcite co-precipitation [70].

Macrophytes dominate mineral regulation between sediment and water [71]. They are capable to remove nutrients even at low loading rates [72]. Their adaptive advantage to lock up minerals from nutrient pool is helpful to check degradation primarily and restore mineral loading later on [47]. $\mathrm{N}$ and $\mathrm{P}$ are intimately related to biological productivity of aquatic ecosystems [37]. The productivity also lies in consonance with developmental stage, ambient nutrient medium and physiognomy of a species [74]. Higher temperature favour bio-production and elevate carbonate driven $\mathrm{pH}$ significantly [75]. The peak nutrient uptake and bioconcentration coincided with peak biomass in summer and autumn [37], [76] and [77]. Substantial OM productions by macrophytes contribute towards nutrient immobilization and provide OC requirement for denitrification while decaying [78]. The upgraded biomass configuration property establishes an additional nutrient and trace element interception feature of hyperaccumulation from the growth medium [79]. Consequently, macrophytes act as unselective nutrient sinks during their peak growth period conferring them rhizofiltration and phytofiltration attributes.

Conclusively, the study discovers that natural setting and homeostasis is influenced by multiple operational factors and pollution conditions. Each site offers a differing dynamics in the trophic ranking of $\mathrm{A} 1>\mathrm{A} 2>\mathrm{D} 2>\mathrm{D} 3>\mathrm{D} 1$ in relation to the type and magnitude of human interference. The structural parts of water, sediment and macrophytes are essential to dissipate the contaminants temporarily to a certain limit. The complications of mismanaged systems are grave as opposed to the managed ones. The study paves way to associate concentration versus speciation connections for diverse pollutants and their on-site phytofiltration options. Thus ecorestoration practices along with retaining optimization capacity can decelerate deterioration.

Acknowledgements. The authors acknowledge Quality Control cum Leaf Tissue Analysis Lab, Sheri Kashmir Agricultural University of Science and Technology (SKAUST), Srinagar-191121; Central Instrumentation Facility (CIF), Pondicherry University- 605014 and Indian Institute of Technology and Management (IITM), Chennai- 600036 for facilitating lab and instrumentation services.

\section{References}

1. Wetzel, R. G. (2001). Land-water interfaces: larger plants. Limnology, 3rd Edn., Academic Press, San Diego.

2. Søndergaard, M., Johansson, L. S., Lauridsen, T. L., JØRGEnSEN, T. B., Liboriussen, L., \& Jeppesen, E. (2010). Submerged macrophytes as indicators of the ecological quality of lakes. Freshwater Biology, 55(4), 893908.

3. Karanlik, S., Agca, N., \& Mehmet, Y. (2011). Spatial distribution of heavy metals content in soils of Amik Plain (Hatay, Turkey). Environmental Monitoring andAssessment,173, 181-191.

4. Zhang, F., Yan, X., Zeng, C., Zhang, M., Shrestha, S., Devkota, L.P., \& Yao, T. (2012). Influence of traffic activity on heavy metal concentrations of roadside farmland soil in mountainous areas. International Journal of Environmental Research and PublicHealth, 9, 1715-1731.

5. EEA. (2012). European Waters - Assessment of Status and Pressures. European Environment Agency, Copenhagen.

6. UNEP. (2007). Global Environment Outlook 4 (GEO-4): environment for development. United Nations Environment Programme, Nairobi, Kenya.

7. Vörösmarty, C.J., McIntyre, P.B., Gessner, M.O., Dudgeon, D., Prusevich, A., Green, P., Glidden, S., Bunn, S.E., Sullivan, C.A., Liermann, C.R., \& Davies, P.M. (2010). Global threats to human water security and river biodiversity. Nature. 467, 555-561. 
8. MEA, Millennium Ecosystem Assessment (2005). Ecosystems and Human Well-Being: Current State and Trends. Island Press, Washington, DC.

9. ANZECC/ARMCANZ. (2000). Australian and New Zealand guidelines for fresh and marine water quality. Australian and New Zealand Environment and Conservation Council and Agriculture and Resource Management Council of Australia and New Zealand, Canberra, 1-103.

10. Batley, G. E. (2000). Implications of the new ANZECC/ARMCANZ water quality guidelines for mining companies, In. Grundon, NJ and Bell, LC. In Proceedings of the Fourth Australian Workshop on Acid Mine Drainage, 221-229.

11. Wright, J. F., Sutcliffe, D. W., \&Furse, M. T. (2000). Assessing the biological quality of fresh waters: RIVPACS and other techniques. In Assessing the biological quality of fresh waters: RIVPACS and other techniques. Freshwater Biological Association.

12. Bunn, S. E., \& Davies, P. M. (2000). Biological processes in running waters and their implications for the assessment of ecological integrity. In Assessing the Ecological Integrity of Running Waters (pp. 61-70). Springer Netherlands.

13. Gupta, A., Ronghang, M., Kumar, P., Mehrotra, I., Kumar, S., Grischek, T., \&Knoeller, K. (2015). Nitrate contamination of riverbank filtrate at Srinagar, Uttarakhand, India: A case of geogenic mineralization. Journal of Hydrology, 531, 626-637.

14. Allan, I., Vrana, B., Greenwood, R., Mills, G., Knutsson, J., Holmberg, A., \&Guigues, N. (2006). Strategic monitoring for the European Water Framework Directive. TrAC, Trends analytical chemistry, 25(7), 704-715.

15. Bierman, P., Lewis, M., Ostendorf, B., \& Tanner, J. (2009). A review of methods for analysing spatial and temporal patterns in coastal water quality. Ecological Indicators, 11 (1), 103-114.

16. Moreno, M., Semprucci, F., Vezzulli, L., Balsamo, M., Fabiano, M., \&Albertelli, G. (2011). The use of nematodes in assessing ecological quality status in the Mediterranean coastal ecosystems. Ecological Indicators, $11(2), 328-336$.

17. APHA,. (2005). APHA, AWWA, \& WEF. Standard methods for the examination of water and wastewater, $21^{\text {st }}$ edn., Washington, DC.

18. Mazej, Z., \& Germ, M. (2009). Trace element accumulation and distribution in four aquatic macrophytes. Chemosphere, 74(5), 642-647.

19. Matusiewicz, H. (2003). Wet digestion methods (Vol. 41). Elsevier: Amsterdam, Netherlands, 193-233.

20. Morford, J.L., Emerson, S.R., Breckel, E.J., \& Kim, S.H. (2005). Diagenesis of oxyanions (V, U, Re, and Mo) in porewaters and sediments from a continental margin. GeochimicaetcosmochimicaActa. 69, 5021 -5032.

21. Parker, J. L., \& Bloom, N. S. (2005). Preservation and storage techniques for low-level aqueous mercury speciation. Science of the Total Environment, 337(1), 253-263.

22. U. S. Environmental Protection Agency. (1994). Method 200.7 : Determination of Metals and Trace Elements in Water and Wastes By Inductively Coupled Plasma - Atomic Emission Spectrometry, Revision 4.4, EMMC.

23. Gupta, P.K. (2004). Soil, Plant, Water and Fertilizer Analysis. Agro Botanica, Vyas Nagar, Bikaner, India.

24. Ryan, J., Estefan, G., \& Rashid, A. (2001). Soil and Plant Analysis Laboratory Manual. International Center for Agricultural Research in the Dry Areas (ICARDA). Aleppo, Syria.

25. Saison, C., Schwartz, C., \& Morel, J. L. (2004). Hyperaccumulation of metals by Thlaspicaerulescens as affected by root development and $\mathrm{Cd}-\mathrm{Zn} / \mathrm{Ca}-\mathrm{Mg}$ interactions. International Journal of phytoremediation, 6(1), 49-61.

26. Boyd, C. E., Tucker, C. S., \&Somridhivej, B. (2016). Alkalinity and hardness: critical but elusive concepts in aquaculture. Journal of the World Aquaculture Society, 47(1), 6-41.

27. Marberly, S.C. (1996). Diel, episodic and seasonal changes in $\mathrm{pH}$ and concentration of inorganic carbon in a productive lake. Freshwater Biology. 35, 579-598.

28. Srebotnjak, T., Carr, G., de Sherbinin, A., \&Rickwood, C. (2012). A global Water Quality Index and hot-deck imputation of missing data. Ecological Indicators, 17, 108-119.

29. Olsen, S., Jeppesen, E., Moss, B., Özkan, K., Beklioğlu, M., Feuchtmayr, H., \& Søndergaard, M. (2014). Factors influencing nitrogen processing in lakes: an experimental approach. Freshwater Biology, 60(4), 646-662.

30. Moiseenko, T. I., Gashkina, N. A., \&Dinu, M. I. (2016). Enrichment of Surface Water by Elements: Effects of Air Pollution, Acidification and Eutrophication. Environmental Processes, 3(1), 39-58.

31. Tchobanoglous, G., Burton F. L., \&Stensel H. D. (2003). Wastewater engineering: treatment and reuse. Metcalf \& Eddy Inc., New York, NY: McGraw Hill.

32. USEPA. (2012). The Assessment and TMDL Tracking and Implementation System. National Summary of State Information. http://iaspub.epa.gov/waters10/attains_nation_cy.control\#total_assessed_waters. 
33. Singh, S. P., \& Singh, B. P. (2010). Geothermal evolution of the evaporite-bearing sequences of the Lesser Himalaya, India. International Journal of Earth Sciences, 99(1), 101-108.

34. Min, K., Kang, H., \& Lee, D. (2011). Effects of ammonium and nitrate additions on carbon mineralization in wetland soils. Soil Biology and Biochemistry, 43(12), 2461-2469.

35. Canavan, R. W., Slomp, C. P., Jourabchi, P., Van Cappellen, P., Laverman, A. M., \& Van den Berg, G. A. (2006). Organic matter mineralization in sediment of a coastal freshwater lake and response to salinization. GeochimicaetCosmochimicaActa, 70(11), 2836-2855.

36. Smolders, A. J. P., Lamers, L. P. M., Lucassen, E. C. H. E. T., Van der Velde, G., \&Roelofs, J. G. M. (2006). Internal eutrophication: how it works and what to do about it - a review. Chemistry and ecology, 22(2), 93-111.

37. Gopal, B. (1990). Nutrient dynamics of aquatic plant communities. In: Ecology and management of aquatic vegetation in the Indian Subcontinent. (pp 177-197). Kluwer Academic Publishers, London.

38. de Jonge, V. N., Elliott, M., \&Orive, E. (2002). Causes, historical development, effects and future challenges of a common environmental problem: eutrophication. Hydrobiologia, 475(1), 1-19.

39. Bu, H., Meng, W., Zhang, Y., \& Wan, J. (2014). Relationships between land use patterns and water quality in the Taizi River basin, China. Ecological Indicators, 41, 187-197.

40. Pandit, A.K. (1999). Freshwater ecosystems of the Himalaya. Parthenon Publishing, New York, London.

41. Thompson, J. B., Schultze-Lam, S., Beveridge, T. J., \& Des Marais, D. J. (1997). Whiting events: biogenic origin due to the photosynthetic activity of cyanobacterial picoplankton. Limnology and oceanography, 42(1), 133-141.

42. Wiik, E., Bennion, H., Sayer, C. D., Davidson, T. A., McGowan, S., Patmore, I. R., \& Clarke, S. J. (2015). Ecological sensitivity of marl lakes to nutrient enrichment: evidence from Hawes Water, UK. Freshwater Biology, 60(11), 2226-2247.

43. Jeelani, G., \& Shah, A. Q. (2006). Geochemical characteristics of water and sediment from the Dal Lake, Kashmir Himalaya: constraints on weathering and anthropogenic activity. Environmental Geology, 50(1), 12-23.

44. BerzasNevado, J. J., Rodríguez Martín-Doimeadios, R. C., Guzmán Bernardo, F. J., Jiménez Moreno, M., Ortega Tardío, S., Sánchez-Herrera Fornieles, M. M., \&Doncel Pérez, A. (2009). Integrated pollution evaluation of the Tagus River in Central Spain. Environmental monitoring and assessment, 156(1), 461-477.

45. Khan, F. A., \& Ansari, A. A. (2005). Eutrophication: an ecological vision. The botanical review, 71(4), 449-482.

46. Yu, S., Yu, G. B., Liu, Y., Li, G. L., Feng, S., Wu, S. C., \& Wong, M. H. (2012). Urbanization impairs surface water quality: eutrophication and metal stress in the Grand Canal of China. River Research and Applications, 28(8), 1135-1148.

47. Kalff, J. (2002). Limnology: inland water ecosystems (Vol. 592). New Jersey: Prentice Hall.

48. Feichtinger, F., Smidt, S., \&Klaghofer, E. (2002). Water and nitrate fluxes at a forest site in the North Tyrolean Limestone Alps. Environmental Science and Pollution Research, 9(2), 31.

49. Hayakawa, A., Ikeda, S., Tsushima, R., Ishikawa, Y., \& Hidaka, S. (2015). Spatial and temporal variations in nutrients in water and riverbed sediments at the mouths of rivers that enter Lake Hachiro, a shallow eutrophic lake in Japan. Catena, 133, 486-494

50. Müller, B., Meyer, J. S., \&Gächter, R. (2016). Alkalinity regulation in calcium carbonate-buffered lakes. Limnology and Oceanography, 61(1), 341-352.

51. Chon, H. S., Ohandja, D. G., \& Voulvoulis, N. (2012). The role of sediments as a source of metals in river catchments. Chemosphere, 88(10), 1250-1256.

52. FAO, Food and agriculture organization. (2010). The wealth of waste: the economics of wastewater use in agriculture. Water Reports, (35).

53. Markich, S. J., Brown, P. L., Batley, G. E., Apte, S. C., \&Stauber, J. L. (2001). Incorporating metal speciation and bioavailability into water quality guidelines for protecting aquatic ecosystems. Australasian Journal of Ecotoxicology, 7(2), 109-122.

54. Downing, J. A., Cole, J. J., Middelburg, J. J., Striegl, R. G., Duarte, C. M., Kortelainen, P., \&Laube, K. A. (2008). Sediment organic carbon burial in agriculturally eutrophic impoundments over the last century. Global Biogeochemical Cycles, 22(1), 1- 10.

55. Wagner, B., Lotter, A. F., Nowaczyk, N., Reed, J. M., Schwalb, A., Sulpizio, R., \&Zanchetta, G. (2009). A 40,000-year record of environmental change from ancient Lake Ohrid (Albania and Macedonia). Journal of Paleolimnology, 41(3), 407-430.

56. Knoll, L. B., Vanni, M. J., Renwick, W. H., \&Kollie, S. (2014). Burial rates and stoichiometry of sedimentary carbon, nitrogen and phosphorus in Midwestern US reservoirs. Freshwater biology, 59(11), 2342-2353. 
57. Schaller, J., Vymazal, J., \&Brackhage, C. (2013). Retention of resources (metals, metalloids and rare earth elements) by autochthonously/allochthonously dominated wetlands: a review. Ecological Engineering, 53, 106114.

58. Ammar, R., Kazpard, V., Wazne, M., El Samrani, A. G., Amacha, N., Saad, Z., \& Chou, L. (2015). Reservoir sediments: a sink or source of chemicals at the surface water-groundwater interface. Environmental monitoring and assessment, $187(9), 579$.

59. Urban, N. R., Brezonik, P. L., Baker, L. A., \& Sherman, L. A. (2009). Sulfate reduction and diffusion in sediments of Little Rock Lake, Wisconsin. Limnology and Oceanography, 39(4), 797-815.

60. Gudasz, C., Bastviken, D., Steger, K., Premke, K., Sobek, S., \& Tranvik, L. J. (2010). Temperature-controlled organic carbon mineralization in lake sediments. Nature, 466(7305), 478-481.

61. de Vicente, I., Guerrero, F., \& Cruz-Pizarro, L. (2010). Chemical composition of wetland sediments as an integrator of trophic state. Aquatic Ecosystem Health 85 Management, 13(1), 99-103.

62. Malmaeus, J. M., \& Rydin, E. (2006). A time-dynamic phosphorus model for the profundal sediments of Lake Erken, Sweden. Aquatic sciences, 68(1), 16-27.

63. Selig, U., \& Schlungbaum, G. (2003). Characterisation and quantification of phosphorus release from profundal bottom sediments in two dimictic lakes during summer stratification. Journal of Limnology. 62(2), 151-162.

64. Garland, J. L., Mackowiak, C. L., \&Zabaloy, M. C. (2010). Organic waste amendment effects on soil microbial activity in a corn-rye rotation: Application of a new approach to community-level physiological profiling. Applied Soil Ecology, 44(3), 262-269.

65. Elser, J. J., Andersen, T., Baron, J. S., Bergström, A. K., Jansson, M., Kyle, M., \& Hessen, D. O. (2009). Shifts in lake N: P stoichiometry and nutrient limitation driven by atmospheric nitrogen deposition. science, 326(5954), 835-837.

66. Abell, J. M., Özkundakci, D., \& Hamilton, D. P. (2010). Nitrogen and phosphorus limitation of phytoplankton growth in New Zealand lakes: implications for eutrophication control. Ecosystems, 13(7), 966-977.

67. Jing, L.D., xi Wu, C., tong Liu, J., guang Wang, H., \&yiAo, H. (2013). The effects of dredging on nitrogen balance in sediment-water microcosms and implications to dredging projects. Ecological Engineering, 52, 167174.

68. Jing, L., Liu, X., Bai, S., Wu, C., Ao, H., \& Liu, J. (2015). Effects of sediment dredging on internal phosphorus: A comparative field study focused on iron and phosphorus forms in sediments. Ecological Engineering, 82, 267271.

69. Turner, A., Millward, G. E., \& Le Roux, S. M. (2004). Significance of oxides and particulate organic matter in controlling trace metal partitioning in a contaminated estuary. Marine Chemistry, 88(3), 179-192.

70. Paramasivam, K., Ramasamy, V., \& Suresh, G. (2015). Impact of sediment characteristics on the heavy metal concentration and their ecological risk level of surface sediments of Vaigairiver, Tamilnadu, India. SpectrochimicaActa Part A: Molecular and Biomolecular Spectroscopy, 137, 397-407.

71. Kissoon, L. T., Jacob, D. L., Hanson, M. A., Herwig, B. R., Bowe, S. E., \&Otte, M. L. (2013). Macrophytes in shallow lakes: Relationships with water, sediment and watershed characteristics. Aquatic Botany, 109, 39-48.

72. Gottschall, N., Boutin, C., Crolla, A., Kinsley, C., \& Champagne, P. (2007). The role of plants in the removal of nutrients at a constructed wetland treating agricultural (dairy) wastewater, Ontario, Canada. Ecological Engineering, 29(2), 154-163.

73. Ismail, Z., Othman, S. Z., Law, K. H., Sulaiman, A. H., \&Hashim, R. (2014). Comparative Performance of Water Hyacinth ( Eichhorniacrassipes) and Water Lettuce (Pistastratiotes) in Preventing Nutrients Build-up in Municipal Wastewater. CLEAN - Soil, Air, Water, 43(4), 521-531.

74. El-Otify, A. M. (2015). Evaluation of the physicochemical and chlorophyll-a conditions of a subtropical aquaculture in Lake Nasser area, Egypt. Beni-Suef University Journal of Basic and Applied Sciences, 4(4), 327337.

75. Hasler, C. T., Butman, D., Jeffrey, J. D., \&Suski, C. D. (2016). Freshwater biota and rising pCO2? Ecology Letters, 19(1), 98-108.

76. Best, E.P.H., Woltman, H. \& Jacobs, F.H.H. (1996). Sediment related growth limitation of Elodea nuttallii as indicated by a fertilization experiment. Freshwater Biology. 36, 33-44.

77. Garbey, C., Murphy, K.J., Thiebaut G. \& Muller, S. (2004). Variation in phosphorus content in aquatic plant tissues offers an efficient tool for determining plant growth strategies along a resource gradient. Fresh Water Biology, 49, 1-11. 
78. McElarney, Y., Rasmussen, P., Foy, R., \& Anderson, N. (2010). Response of aquatic macrophytes in Northern Irish softwater lakes to forestry management; eutrophication and dissolved organic carbon. Aquatic Botany, 93(4), 227-236.

79. Shaltout, K. H., Galal, T. M., \& El-Komi, T. M. (2014). Biomass, nutrients and nutritive value of PersicariasalicifoliaWilld.in the water courses of Nile Delta, Egypt. RendicontiLincei, 25(2), 167-179. 